\title{
Retinitis pigmentosa-associated rhodopsin mutant T17M induces endoplasmic reticulum (ER) stress and sensitizes cells to ER stress-induced cell death
}

\author{
HAIBO JIANG, SIQI XIONG and XIAOBO XIA \\ Department of Ophthalmology, Xiangya Hospital, Central South University, Changsha, Hunan 410078, P.R. China
}

Received August 15, 2013; Accepted February 14, 2014

DOI: $10.3892 / \mathrm{mmr} .2014 .1987$

\begin{abstract}
Retinitis pigmentosa (RP) is a group of inherited diseases that primarily affect light-sensitive rods and cones in the retina. Rhodopsin mutations, including the $\mathrm{T} 17 \mathrm{M}$ mutation, are associated with the autosomal dominant form of retinitis pigmentosa (ADRP) and have been linked to abnormal protein folding. However, the molecular mechanisms underlying $\mathrm{T} 17 \mathrm{M}$ rhodopsin-induced retinal degeneration are yet to be elucidated. In the present study, Human embryonic kidney (HEK) 293 and ARPE-19 cells were transfected with myc-tagged wild-type (WT) and T17M rhodopsin constructs. Cells were fixed and stained with anti-myc antibodies and the localization of WT and T17M rhodopsin was visualized using immunofluorescence microscopy. Turnover rates of WT and T17M rhodopsin were measured using western blot analysis. In addition, endoplasmic reticulum (ER) stress-induced cell death was analyzed in WT and T17M rhodopsin-transfected cells using nuclear staining. Misfolded T17M rhodopsin was observed to be abnormally localized in the ER, while WT rhodopsin was predominantly found at the plasma membrane. Protein turnover analysis revealed that T17M rhodopsin was more rapidly degraded by proteasomes than WT rhodopsin. Furthermore, overexpression of T17M rhodopsin was observed to induce cell death and increase cytotoxicity; predisposing cells to ER stress-induced cell death. These findings show novel insight into the properties of T17M rhodopsin and highlight the role of ER stress in T17M-associated RP.
\end{abstract}

\section{Introduction}

Retinitis pigmentosa (RP) is a rare, inherited disease, which is caused by the progressive loss of rod and cone photoreceptors. The clinical hallmarks of RP include sensitivity to dim light, abnormal visual function and characteristic bone spicule deposits of pigment in the retina (1). RP has been associated

Correspondence to: Professor Xiaobo Xia, Department of Ophthalmology, Xiangya Hospital, Central South University, 87 Xiangya Road, Changsha, Hunan 410078, P.R. China

E-mail: xbxia21@163.com

Key words: retinitis pigmentosa, rhodopsin, endoplasmic reticulum stress, misfolding with mutations in $>45$ genes, a number of which affect proteins expressed exclusively in rod photoreceptor cells, including the $\alpha$ and $\beta$ subunits of the rod cyclic guanosine monophosphate (cGMP) phosphodiesterase, the rod cGMP-gated channel and rhodopsin (2). Rhodopsin is a photon receptor and light-sensitive visual pigment, which initiates phototransduction. Autosomal dominant retinitis pigmentosa (ADRP) is associated with mutations in rhodopsin (2). At present, >140 RP-associated rhodopsin mutations have been identified (3). The human rhodopsin gene encodes a 348-amino acid protein that has seven transmembrane domains, with a luminal $\mathrm{N}$ terminus and a cytoplasmic $\mathrm{C}$ terminus (4). Dominant rhodopsin mutations are divided into class I, II and III $(5,6)$. T17M rhodopsin is a class II rhodopsin mutant and it lacks the capacity to form the normal rhodopsin chromophore with 11-cis-retinal due to a defect in thermal stability folding (7). Patients carrying the T17M rhodopsin mutant have altitudinal visual field defects, associated with less impaired rod and cone functions in the inferior than in the superior field $(8,9)$. Expression of the human T17M mutant rhodopsin transgene in mice has been associated with photoreceptor apoptosis in response to moderate exposure to light. However, this phenotype was not observed in non-transgenic littermates or in mice expressing the human $\mathrm{P} 28 \mathrm{H}$ mutant rhodopsin transgene. This finding suggests that the T17M mutation abolishes glycosylation at the Asn-15 site of rhodopsin and that this elimination of glycosylation is associated with an increased sensitivity to light-induced damage (10).

The present study aimed to investigate the differences in the localization and degradation of RP-associated T17M mutant rhodopsin compared with wild-type (WT) rhodopsin, elucidate the role of endoplasmic reticulum (ER) stress and identify potential candidates for therapy.

\section{Materials and methods}

Cell culture, plasmid constructs and transfection. Human embryonic kidney 293 (HEK-293) and ARPE-19 retinal pigmented epithelium cells were obtained from American Type Culture Collection (Rockville, MD, USA) and maintained according to the providers instructions. HEK-293 and ARPE-19 cells were cultured in Dulbecco's modified Eagle's medium (DMEM) and DMEM/F-12, respectively, supplemented with $10 \%$ fetal bovine serum and $50 \mu \mathrm{g} / \mathrm{ml}$ penicillin-streptomycin at $37^{\circ} \mathrm{C}$ and $5 \% \mathrm{CO}_{2}$. The coding 
sequence of the rhodopsin gene was amplified from a human cDNA library (Invitrogen Life Technologies, Carlsbad, CA, USA) using LA Taq DNA polymerase (Takara Bio Inc., Shiga, Japan). The fragment was ligated into the mammalian expression vector pcDNA $^{\mathrm{TM}} 3.1 /$ myc HisA(+) (Invitrogen Life Technologies) and transformed into competent Escherichia coli DH5 $\alpha$ cells according to the manufacturer's instructions. The integrity of all inserts was determined using direct sequence analysis and transfections were performed using Lipofectamine ${ }^{\circledR} 2000$ (Invitrogen Life Technologies) according to the manufacturer's instructions. In brief, cells were cultured in 12-well plates in serum-free DMEM supplemented with $1.6 \mu \mathrm{g}$ DNA and $4 \mu \mathrm{l}$ Lipofectamine 2000 for $4 \mathrm{~h}$. The transfection media was then replaced and incubated with fresh standard HEK-293 media.

Immunofluorescence microscopy. Cells expressing WT and $\mathrm{T} 17 \mathrm{M}$ rhodopsin were cultured on glass coverslips for 24-48 h. Cells were then fixed using 4\% paraformaldehyde for $10 \mathrm{~min}$ and permeabilized using $0.1 \%$ Triton $\mathrm{X}-100$ in phosphate-buffered saline (PBS) for $10 \mathrm{~min}$ at $25^{\circ} \mathrm{C}$. Subsequent to blocking with $3 \%$ bovine serum albumin for $30 \mathrm{~min}$, cells were incubated with mouse anti-myc (dilution, 1:300) and rabbit anti-protein disulfide isomerase (PDI) (dilution, 1:300) antibodies (Sigma-Aldrich, St. Louis, MO, USA). Cells were then incubated with Alexa Fluor ${ }^{\circledR}$ 488-labeled goat anti-mouse IgG and Alexa Fluor ${ }^{\circledR}$ 549-labeled goat anti-rabbit IgG antibodies (dilution, 1:400; Invitrogen Life Technologies). Cell nuclei were counter-stained using DAPI (Invitrogen Life Technologies). Images were captured using a Leica microscope (Leica Microsystems, Wetzlar, Germany) with appropriate excitation and emission filter pairs.

Western blot analysis. For the degradation experiment, cells were treated with or without $10 \mu \mathrm{M}$ proteasome inhibitor MG132 for $12 \mathrm{~h}$ (Sigma Aldrich). For the turnover rate experiment, ARPE-19 cells were treated with $50 \mu \mathrm{g} / \mathrm{ml}$ cycloheximide for $0,2,4$ or $6 \mathrm{~h}$. (Sigma Aldrich). Subsequently, the cells were lysed in 2X SDS sample buffer (63 mM Tris $\mathrm{HCl}, 10 \%$ glycerol and $2 \%$ SDS). The supernatant was collected and the protein concentration was determined using a Pierce ${ }^{\mathrm{TM}}$ protein assay kit (Pierce Chemical Company, Rockford, IL, USA). Protein extracts $(30 \mu \mathrm{g})$ were separated using SDS-PAGE and were then transferred to polyvinylidene fluoride membranes (Millipore Corporation, Billerica, MA, USA). Membranes were incubated for $1 \mathrm{~h}$ in blocking solution (5\% dry milk in $0.1 \%$ Triton $\mathrm{X}-100 / \mathrm{PBS}$ ), followed by incubation with mouse anti-myc (dilution, 1:5,000; Sigma-Aldrich), mouse anti-binding immunoglobulin protein (BiP; dilution, 1:1,000; Sigma-Aldrich) and mouse anti-actin (dilution, 1:10,000; Sigma-Aldrich) antibodies in blocking solution. Subsequent to being washed with $0.1 \%$ Triton X-100/PBS buffer, membranes were incubated with goat anti-mouse IgG antibody conjugated to horseradish peroxidase (dilution, 1:10,000; Invitrogen Life Technologies) for $1 \mathrm{~h}$ and visualized using an enhanced chemiluminescence kit according to the manufacturer's instructions (GE Healthcare, Little Chalfont, UK).

Cell death detection. Following a pre-coat with $50 \mu \mathrm{g} / \mathrm{ml}$ poly-L-lysine, HEK293 cells were transfected with myc-tagged
WT or T17M mutant vectors. A total of $20 \mathrm{~h}$ after transfection, cells were treated with dimethylsulfoxide (DMSO; Sigma-Aldrich), $2 \mathrm{mg} / \mathrm{ml}$ tunicamycin for $8 \mathrm{~h}$, or $100 \mu \mathrm{M}$ ROS scavengers butylated hydroxyanisole (BHA; Sigma-Aldrich) and $1 \mathrm{mM} \mathrm{N}$-acetylcysteine (NAC; Sigma-Aldrich) for $12 \mathrm{~h}$, respectively. Cell death was then quantified by counting the cells that exhibited highly condensed and/or fragmented DNA subsequent to staining with Hoechst 33342 nuclear dye. At least 1,000 cells were counted per well.

ROS detection. Cells were digested with $0.125 \%$ trypsin, pelleted and suspended in medium containing $20 \mu \mathrm{M}$ dichloro-dihydro-fluorescein diacetate (Sigma-Aldrich). After incubation for $30 \mathrm{~min}$, cells were centrifuged at $2000 \mathrm{x} \mathrm{g}$ for $10 \mathrm{~min}$, resuspended in fresh medium and subjected to fluorescence-activated cell sorting analysis using the MoFLo ${ }^{\mathrm{TM}} \mathrm{XDP}$ cell sorter (Beckman Coulter, Inc., Brea, CA, USA). Data were analyzed using FlowJo software (Tree Star, Inc., Ashland, OR, USA) in order to assess the mean fluorescence intensity. Data are presented relative to the control cells.

Statistical analysis. Data represent quantification of three independent experiments. Results are presented as the mean \pm standard deviation. One way analysis of variance followed by Tukey or Dunnett's tests were performed using GraphPad Prism 5 software (GraphPad Software, San Diego California USA). $\mathrm{P}<0.05$ was considered to indicate a statistically significant difference.

\section{Results}

$R P$-associated T17M mutant rhodopsin is translocated to the $E R$. In order to investigate whether $\mathrm{WT}$ and $\mathrm{T} 17 \mathrm{M}$ rhodopsin have a similar localization, myc-tagged WT and T17M proteins were heterologously expressed in ARPE-19 cells and the proteins were visualized using immunofluorescence microscopy. Following transient transfection, double immunofluorescence staining revealed that the $\mathrm{T} 17 \mathrm{M}$ rhodopsin mutant protein was localized in the ER, as evidenced by the strong co-localization with the ER marker PDI (Fig. 1). By contrast, WT rhodopsin was observed to be localized at the cell membrane and did not co-localize with PDI (Fig. 1).

T17M mutant rhodopsin is unstable and degraded by proteasomes. It was examined whether accumulation of the T17M protein in HEK293 cells was sensitive to proteasome inhibition, which is a characteristic of ER-associated protein degradation (ERAD) substrates. Cells were treated with or without the proteasome inhibitor MG132 (10 $\mu \mathrm{M}$ for $12 \mathrm{~h})$ and the accumulation of transiently expressed myc-tagged WT and T17M rhodopsin proteins were compared in HEK293 cells. The levels of WT and T17M rhodopsin were observed to markedly increase following MG132 treatment. However, the aggregation of rhodopsin was observed to be higher in the T17M rhodopsin-transfected cells than in the WT rhodopsin-transfected cells following MG132 treatment (Fig. 2).

The turnover rates of the myc-tagged rhodopsin proteins were assessed in ARPE-19 cells, which were treated with $50 \mu \mathrm{g} / \mathrm{ml}$ cycloheximide for various time-periods (Fig. 3A). $\mathrm{T} 17 \mathrm{M}$ rhodopsin exhibited a more rapid turnover than WT 

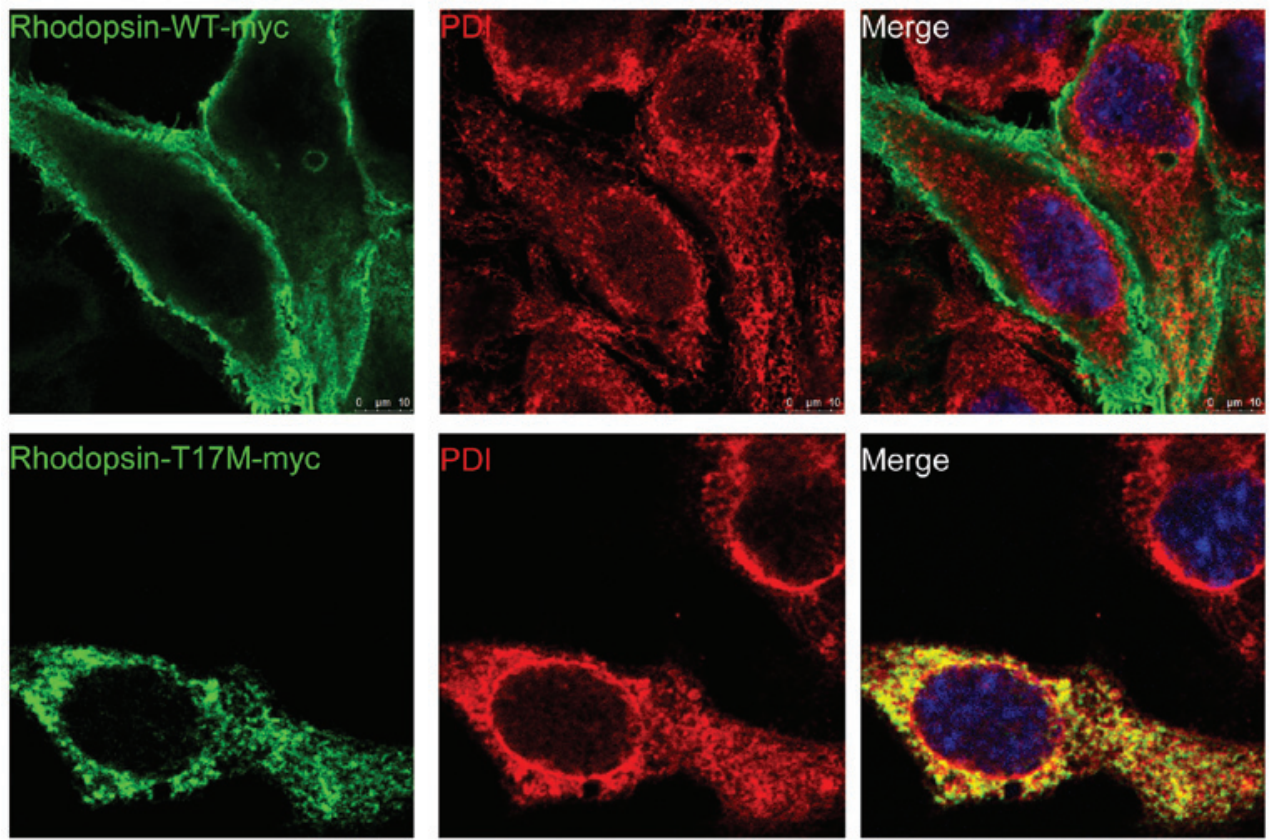

Figure 1. Subcellular localization of WT and T17M mutant rhodopsin in transiently transfected ARPE-19 cells detected using immunofluorescence microscopy. ARPE-19 cells were transfected with myc-tagged WT or T17M mutant rhodopsin (green) and visualized using immunofluorescence microscopy. Cells were co-stained with the endoplasmic reticulum marker PDI (red). Nuclei were stained with DAPI (blue). Scale bar, $10 \mu \mathrm{m}$. WT, wild-type; PDI, protein disulfide isomerase.
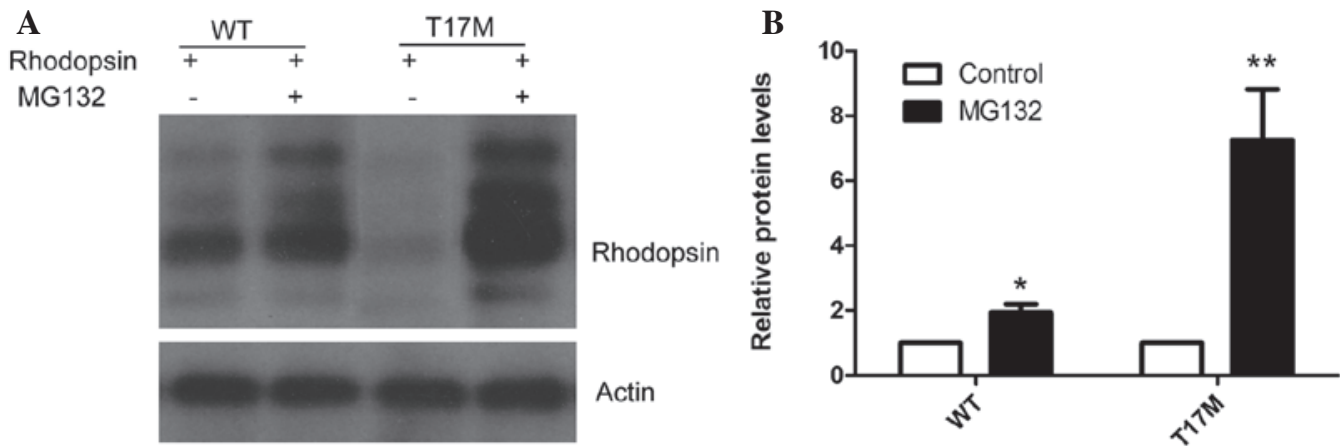

Figure 2. Proteasome inhibition and rhodopsin protein levels. (A) HEK293 cells were transfected with either myc-tagged WT rhodopsin or T17M mutant rhodopsin constructs. A total of $20 \mathrm{~h}$ after transfection, cells were treated with or without $10 \mu \mathrm{M}$ MG132 for $12 \mathrm{~h}$. The rhodopsin proteins were detected using western blot analysis with anti-myc and -actin antibodies. Actin was used as a loading control. (B) Quantification of the relative changes in rhodopsin construct protein expression prior and subsequent to MG132 treatment. Data are presented as the mean \pm standard deviation of three independent experiments. ${ }^{*} \mathrm{P}<0.05$ and ${ }^{* *} \mathrm{P}<0.01$ vs. control. WT, wild-type; HEK293, human embryonic kidney 293.

A

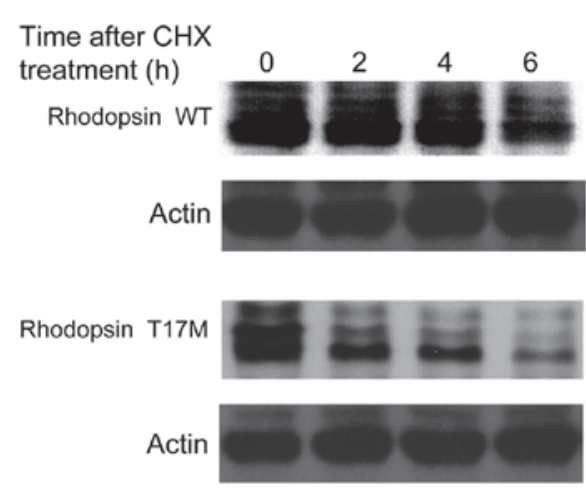

B

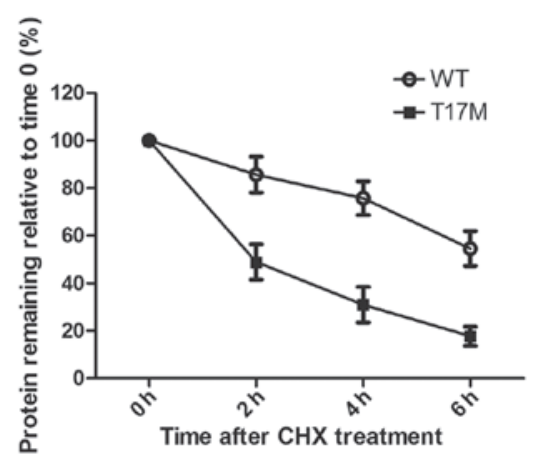

Figure 3. Turnover of rhodopsin. (A) ARPE-19 cells were transfected with either myc-tagged WT or T17M mutant rhodopsin. A total of $20 \mathrm{~h}$ after transfection, cells were treated with CHX and the lysates were collected at the indicated time-points. Western blot analysis of rhodopsin and actin was performed using the cell lysates. (B) Quantification of rhodopsin protein levels relative to time-point zero. T17M mutant rhodopsin exhibited a more rapid degradation than WT rhodopsin. The graph represents an average of three experiments. Data are presented as the mean \pm standard deviation. CHX, cycloheximide; WT, wild-type. 
A

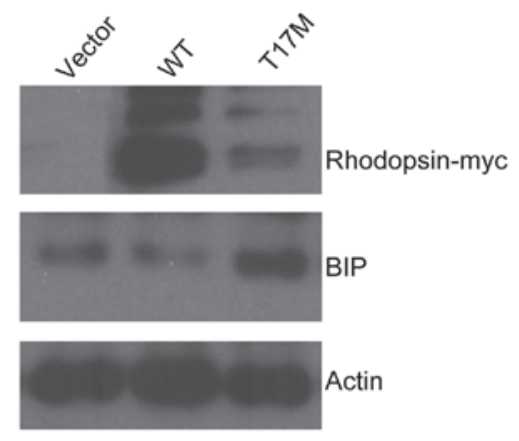

B

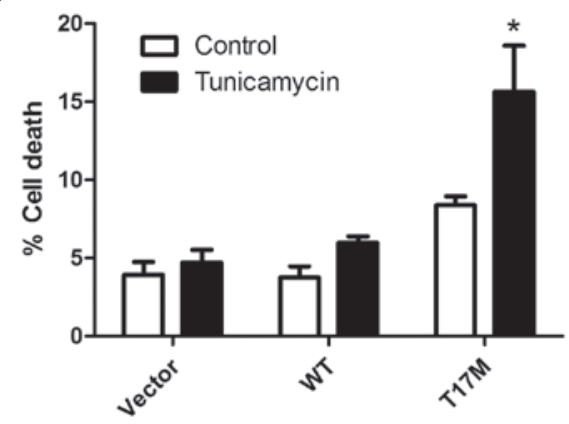

Figure 4. T17M mutant rhodopsin sensitizes cells to ER stress-induced cell death. (A) Activation of the UPR in HEK293 cells transfected with myc-tagged T17M rhodopsin constructs. Western blot analysis was performed using equal quantities of cell lysates and antibodies against the UPR marker BiP, as well as rhodopsin and actin. (B) HEK293 cells were transfected with an empty vector or the indicated myc-tagged rhodopsin constructs. A total of $20 \mathrm{~h}$ after transfection, cells were treated with tunicamycin or dimethylsulfoxide (control) at a final concentration of $2 \mathrm{mg} / \mathrm{ml}$ for $8 \mathrm{~h}$. Cell death was then quantified by counting the cells that had highly condensed and/or fragmented DNA following cell staining with the nuclear dye Hoechst 33342. T17M mutant rhodopsin-transfected cells exhibit a significant increase in cell death compared with those transfected with an empty vector or WT rhodopsin with or without tunicamycin treatment The graph represents an average of three independent experiments and the data are shown as the mean \pm standard deviation. ${ }^{*} \mathrm{P}<0.05$ vs. vector, WT and T17M control cells. WT, wild-type; ER, endoplasmic reticulum; BiP, binding immunoglobulin protein; UPR, unfolded protein response; HEK293, human embryonic kidney 293.

A

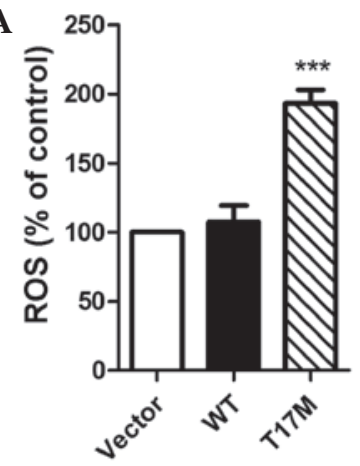

B

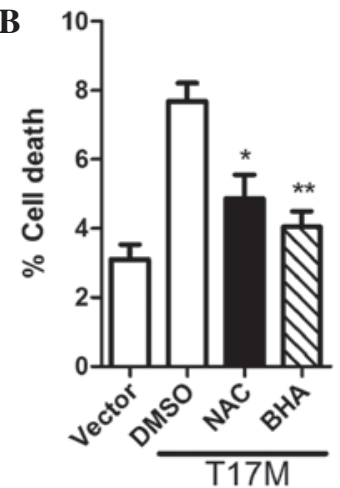

Figure 5. T17M rhodopsin overexpression-induced cell death is decreased with ROS scavengers. (A) Relative levels of intracellular ROS were detected in control cells (vector) and cells expressing either WT or T17M rhodopsin. (B) T17M rhodopsin-induced HEK293 cell death was partially inhibited with the ROS scavengers NAC and BHA. ${ }^{*} \mathrm{P}<0.05$ and ${ }^{* *} \mathrm{P}<0.01$ vs. DMSO; ${ }^{* * *} \mathrm{P}<0.01$ vs. vector and WT. ROS, reactive oxygen species; WT, wild-type; DMSO, dimethylsulfoxide; NAC, $N$-acetylcysteine; BHA, butylated hydroxyanisole.

rhodopsin, with a half-life of $2 \mathrm{~h}$ compared with $6 \mathrm{~h}$, respectively (Fig. 3B).

T17M mutant rhodopsin activates ER-stress and sensitizes cells to ER stress-induced cell death. To determine whether the accumulation of T17M in the ER activates ER stress, the expression of the resident ER chaperone BiP, which is an unfolded protein response marker, was assessed in WT and $\mathrm{T} 17 \mathrm{M}$ rhodopsin-transfected cells. BiP was observed to be upregulated in cells transfected with rhodopsin $\mathrm{T} 17 \mathrm{M}$ constructs, indicating that the ER stress response was activated (Fig. 4A). Prolonged ER stress caused by the accumulation of unfolded proteins in the ER leads to cell death; therefore, WT and misfolded rhodopsin proteins were overexpressed in HEK293 cells and the effect on cell death was assessed. HEK293 cells were transfected with myc-tagged WT and T17M rhodopsin constructs and empty vectors. Cell death was then quantified in the absence or presence of $2 \mathrm{mg} / \mathrm{ml}$ tunicamycin, which is an ER stress inducer. Cells transfected with

the T17M rhodopsin mutant were found to be more sensitive to ER stress-induced cell death than those transfected with WT rhodopsin or empty vectors. Moreover, an increase in cell death was observed in the cells exogenously expressing T17M rhodopsin (Fig. 4B).

ROS scavengers protect against T17M rhodopsin-induced cell death. To determine whether ROS production contributes to T17M rhodopsin-induced cell death, cells expressing T17M rhodopsin were treated with the ROS scavengers BHA $(100 \mu \mathrm{M})$ and NAC $(1 \mathrm{mM})$. Subsequent to $12 \mathrm{~h}$ of treatment, cell death was assessed using Hoechst 33342 staining. ROS levels were observed to be significantly increased in the cells transfected with T17M rhodopsin compared with those transfected with the empty vector or WT rhodopsin (Fig. 5A). Furthermore, cell death was found to be significantly decreased in the cells treated with BHA and NAC compared with those treated with DMSO (Fig. 5B). These findings suggest that T17M rhodopsin induces cell death, at least in part, through a ROS-mediated pathway.

\section{Discussion}

In the present study, human T17M mutant rhodopsin was found to be abnormally localized in the ER. Furthermore, misfolded T17M mutant rhodopsin was more rapidly degraded by proteasomes than WT rhodopsin. Overexpression of T17M rhodopsin was found to increase cytotoxicity and predispose cells to ER stress-induced cell death. Moreover, the cell death induced by T17M rhodopsin overexpression was observed to be decreased with ROS scavenger treatment.

Rhodopsin is a G-protein-coupled receptor that mediates light-induced signal transduction in rod photoreceptor cells (11) and is glycosylated and transported to the plasma membrane (12). Heterozygous rhodopsin mutations which cause amino acid substitutions have been reported to cause human ADRP (6). It has also been reported that transgenic animals expressing a mutant rhodopsin gene demonstrate retinal degeneration $(7,13,14)$. Mutant rhodopsin phenotypes are divided into three classes: class I, II and III. Class I mutants 
are capable of forming normal rhodopsin chromophores with 11-cis-retinal and are trafficked to the plasma membrane. However, their transducing activation is inefficient upon illumination. Class III mutants are only capable of forming a few rhodopsin chromophores, due to their low expression levels. Moreover, class III mutants that accumulate in the ER exhibit high mannose glycosylation (5). Class II mutants accumulate in the ER and are incapable of forming the chromophore due to the inability to bind with 11-cis-retinal (7). The rhodopsin T17M mutation is an example of a class II mutant and has a defect in protein folding (15). The substitution of threonine to methionine at position 17 in the T17M rhodopsin mutant, has been proposed to affect protein folding due to the proximity of the substitution to the N2-N15 glycosylation site (16). The present study confirmed that the rhodopsin T17M mutant, which is involved in RP, was localized in the ER and was incapable of plasma membrane trafficking. This retention of the misfolded protein in the ER supports the pathogenicity of RP mutations at a cellular level.

The pathogenesis of neurodegeneration is often associated with disturbances in protein folding, aggregation and degradation (17). Accurate protein folding and processing are required to maintain cellular homeostasis (18). Protein misfolding not only affects protein function, but leads to the formation of potentially toxic aggregates (19). Cells have complex systems in order to eliminate unwanted and potentially toxic proteins. Incorrectly folded rhodopsin is detected by specialized ER sensors and is either subject to additional folding cycles or degradation. In T17M rhodopsin transgene mice, no significant difference was observed in ERAD gene expression compared with the control; however, the autophagy degradation pathway was observed to be upregulated (20). In the present study, proteasomal inhibition and analysis of protein turnover revealed that the T17M mutant protein has an accelerated rate of degradation compared with the WT protein. Furthermore, the proteasome was found to be responsible for this increased protein turnover. It has been reported that some ER-associated degradation is proteasome-independent (21). Therefore, the mechanism underlying the degradation of the T17M mutant requires further investigation.

The accumulation of misfolded proteins in the ER induces ER stress and has been implicated in human diseases, including neurodegenerative diseases $(22,23)$. In the present study, it was hypothesized that ER stress induced by T17M rhodopsin may have a role in the development of RP due to three reasons. Firstly, the T17M rhodopsin mutant was observed to be localized in the ER. Secondly, the presence of the T17M mutant in the ER was found to increase cell death following exposure to the ER stressor tunicamycin, suggesting that the misfolded T17M rhodopsin protein negatively impacts ER homeostasis, thereby increasing the susceptibility of cells to ER stress-induced cell death. Of note, ER stress-induced retinal pathology in rhodopsin $\mathrm{P} 23 \mathrm{H}$-expressing rats has been observed to be reduced by BiP protein (13). Therefore, increased expression of $\mathrm{BiP}$ or other chaperones may reduce T17M-induced ER stress. The rhodopsin T17M mutant has been reported to exhibit a reversible folding capacity (15) and is transported partially to the rod outer segments in transgenic Xenopus photoreceptor neurons (16). Thus, small pharmacological molecule chaperones that correct folding-deficient rhodopsin may be potential therapeutic options. Thirdly, activation of the ER stress response has been reported to lead to changes in intracellular $\mathrm{Ca}^{2+}$ concentration (24), as well as the release of ROS from protein folding chaperones involved in disulphide bond formation (25). Chaperones, including $\mathrm{BiP}$ require energy to function. ER stress therefore increases the demand on ATP synthesis by the mitochondria. Thus, it is possible that ER stress may induce other downstream effects and may cause a cascade of damage, leading to cell death $(26,27)$. Moreover, in the present study, ROS scavengers were found to reduce T17M rhodopsin-induced cell death. This study identifies a mechanism of RP pathogenesis induced by rhodopsin T17M mutant and provides a potential treatment strategy of the disease.

\section{Acknowledgements}

This paper was supported by the grant from the National Natural Science Foundation of China (No. 81170844).

\section{References}

1. Dryja TP, McGee TL, Hahn LB, et al: Mutations within the rhodopsin gene in patients with autosomal dominant retinitis pigmentosa. N Engl J Med 323: 1302-1307, 1990.

2. Hartong DT, Berson EL and Dryja TP: Retinitis pigmentosa. Lancet 368: 1795-1809, 2006

3. Mendes HF, van der Spuy J, Chapple JP and Cheetham ME: Mechanisms of cell death in rhodopsin retinitis pigmentosa: implications for therapy. Trends Mol Med 11: 177-185, 2005.

4. Nathans J and Hogness DS: Isolation and nucleotide sequence of the gene encoding human rhodopsin. Proc Natl Acad Sci USA 81: 4851-4855, 1984.

5. Kaushal S and Khorana HG: Structure and function in rhodopsin 7. Point mutations associated with autosomal dominant retinitis pigmentosa. Biochemistry 33: 6121-6128, 1994.

6. Sung CH, Davenport CM and Nathans J: Rhodopsin mutations responsible for autosomal dominant retinitis pigmentosa. Clustering of functional classes along the polypeptide chain. J Biol Chem 268: 26645-26649, 1993.

7. Li T, Sandberg MA, Pawlyk BS, et al: Effect of vitamin A supplementation on rhodopsin mutants threonine-17 --> methionine and proline-347 --> serine in transgenic mice and in cell cultures. Proc Natl Acad Sci USA 95: 11933-11938, 1998.

8. Jacobson SG, Kemp CM, Sung CH and Nathans J: Retinal function and rhodopsin levels in autosomal dominant retinitis pigmentosa with rhodopsin mutations. Am J Ophthalmol 112: 256-271, 1991.

9. Sung CH, Davenport CM, Hennessey JC, et al: Rhodopsin mutations in autosomal dominant retinitis pigmentosa. Proc Natl Acad Sci USA 88: 6481-6485, 1991.

10. White DA, Fritz JJ, Hauswirth WW, Kaushal S and Lewin AS: Increased sensitivity to light-induced damage in a mouse model of autosomal dominant retinal disease. Invest Ophthalmol Vis Sci 48: 1942-1951, 2007.

11. Ridge KD, Abdulaev NG, Sousa $M$ and Palczewski $K$ : Phototransduction: crystal clear. Trends Biochem Sci 28: 479-487, 2003.

12. Chapple JP, Grayson C, Hardcastle AJ, Saliba RS, van der Spuy J and Cheetham ME: Unfolding retinal dystrophies: a role for molecular chaperones? Trends Mol Med 7: 414-421, 2001.

13. Gorbatyuk MS, Knox T, LaVail MM, et al: Restoration of visual function in $\mathrm{P} 23 \mathrm{H}$ rhodopsin transgenic rats by gene delivery of BiP/Grp78. Proc Natl Acad Sci USA 107: 5961-5966, 2010.

14. Krebs MP, White DA and Kaushal S: Biphasic photoreceptor degeneration induced by light in a T17M rhodopsin mouse model of cone bystander damage. Invest Ophthalmol Vis Sci 50: 2956-2965, 2009.

15. Krebs MP, Holden DC, Joshi P, Clark CL III, Lee AH and Kaushal S: Molecular mechanisms of rhodopsin retinitis pigmentosa and the efficacy of pharmacological rescue. J Mol Biol 395: 1063-1078, 2010. 
16. Tam BM and Moritz OL: The role of rhodopsin glycosylation in protein folding, trafficking, and light-sensitive retinal degeneration. J Neurosci 29: 15145-15154, 2009.

17. Selkoe DJ: Cell biology of protein misfolding: The examples of Alzheimer's and Parkinson's diseases. Nat Cell Biol 6: 1054-1061, 2004.

18. Wickner S, Maurizi MR and Gottesman S: Posttranslational quality control: folding, refolding, and degrading proteins. Science 286: 1888-1893, 1999.

19. Chaudhuri TK and Paul S: Protein-misfolding diseases and chaperone-based therapeutic approaches. FEBS J 273: 1331-1349, 2006.

20. Kunte MM, Choudhury S, Manheim JF, et al: ER stress is involved in T17M rhodopsin-induced retinal degeneration. Invest Ophthalmol Vis Sci 53: 3792-3800, 2012.

21. Finger A, Knop M and Wolf DH: Analysis of two mutated vacuolar proteins reveals a degradation pathway in the endoplasmic reticulum or a related compartment of yeast. Eur J Biochem 218: 565-574, 1993.
22. Zhao L and Ackerman SL: Endoplasmic reticulum stress in health and disease. Curr Opin Cell Biol 18: 444-452, 2006.

23. Griciuc A, Aron L and Ueffing M: ER stress in retinal degeneration: a target for rational therapy? Trends Mol Med 17: 442-451, 2011.

24. Scorrano L, Oakes SA, Opferman JT, et al: BAX and BAK regulation of endoplasmic reticulum $\mathrm{Ca}^{2+}$ : a control point for apoptosis. Science 300: 135-139, 2003.

25. Haynes CM, Titus EA and Cooper AA: Degradation of misfolded proteins prevents ER-derived oxidative stress and cell death. Mol Cell 15: 767-776, 2004.

26. Stefani IC, Wright D, Polizzi KM and Kontoravdi C: The role of ER stress-induced apoptosis in neurodegeneration. Curr Alzheimer Res 9: 373-387, 2012

27. Hayashi T, Saito A, Okuno S, Ferrand-Drake M, Dodd RL and Chan PH: Damage to the endoplasmic reticulum and activation of apoptotic machinery by oxidative stress in ischemic neurons. J Cereb Blood Flow Metab 25: 41-53, 2005. 